\title{
Minimal Invasive Anterior Decompression in Tuberculosis of Thoracolumbar Junction of the Spine - Experience With SynFrame
}

\author{
Karn NK, Rao BS, ${ }^{2}$ Prabhakar $M M^{2}$ \\ 'Department of Orthopaedics, BP Koirala Institute of Health Sciences, Dharan, Nepal, ${ }^{2}$ Department of Orthopedics, Paraplegia \\ Hospital, Ahmedabad, Gujrat, India.
}

\section{ABSTRACT}

Introduction: This study assesses the role of new retractor system SynFrame for anterior decompression of tuberculosis of thoracolumbar junction of the spine.

Methods: This study includes five consecutive patients with tuberculosis of thoracolumbar junction treated with minimal invasive anterior decompression using a new table mounted retractor system SynFrame (Stratec Medical, Switzerland). The thoracolumbar junction was approached by a left sided thoracotomy. Short construct pedicle screw stabilization was done in all cases before decompression. The anterior column was reconstructed using expandable cage $(n=3)$ and autologous tricortical graft $(n=2)$.

Results: The mean operating time was 100 minutes (range 90-120). Mean overall blood loss was 400 $\mathrm{ml}$. No operation had to change into an open procedure. There were neither intra nor postoperative complications related to minimal access in particular, nor visceral/vascular complications.

Conclusions: The ring retractor system allows minimal open surgery to the spine by carrying different devices as well as endoscope, providing an excellent visualization of the operating field, and is safe and easy to use. The only disadvantage is its high cost.

Key Words: anterior decompression, minimal invasive spine surgery, SynFrame

\section{INTRODUCTION}

Minimal invasive approaches to the thoracic and lumbar spine were introduced in the early 1990 s. ${ }^{1,2}$ The main goal of these efforts had been the reduction of surgical trauma, since anterior open approaches to thoracic or lumbar spine were associated with significant complication rate. ${ }^{3,4}$ Major patients' complaints were intercostal neuralgia and post-thoracotomy pain syndromes. ${ }^{3,5}$ Already the first reports using minimal invasive technology for spine interventions have demonstrated a reduction of the access morbidity, reduced intraoperative blood loss, fewer days in intensive care and an overall reduction in the hospital stay. ${ }^{1,2,6,7}$

A variety of minimal surgery access strategies to the thoracic and lumbar spine have been developed in recent years, named either minimal invasive spinal surgery (MISS), video-assisted thoracoscopic surgery (VATS), laparoscopic-assisted spinal surgery or retroperitoneal endoscopic surgery. ${ }^{2,6,8-10}$ 
Karn et al. Minimal Invasive Anterior Decompression in Tuberculosis of ...

This study was done to see the role of new retractor system SynFrame ${ }^{11}$ for anterior decompression of tuberculosis of thoracolumbar junction of the spine.

\section{METHODS}

The prospective interventional study was carried out in Paraplegia Hospital, Ahmedabad, India between 2007 and 2008. Ethical approval and patient consent was taken. Five consecutive patients with tuberculosis of thoracolumbar junction of the spine with neurological deficit, abscess or kyphosis more than 15 degree were operated in two stages. In the first stage, fixation of the spine was done with short construct pedicle screw and in the second stage, anterior decompression, partial corpectomy and insertion of expandable cage/tricortical bone graft with corticocancellous bone graft for fusion to restore intervertebral height and axial support was done. All patients were taking standard anti-tubercular therapy.

The retractor system SynFrame (Stratec Medical, Switzerland) is a stable and adjustable ring system. It is fixed sterile by two adjustable arms onto the operating table, allowing a $360^{\circ}$ surgical access from any point inside the ring. ${ }^{11}$ One of the major advantage of the system is that it provides permanent stability of the operative field, enabling the surgeon to perform small access to the spine without further manipulation. The blades are clicked onto the ring and can be located at any point on the ring, depending on the requirement of the surgeon. In all of our cases, adjustable retractor blades were used; although the SynFrame system also offers the use of modified Hohmann levers. ${ }^{11}$ The blades can be adjusted individually in all three planes due to their connections to the ring by a clamp. Different blade lengths, from 60 to $160 \mathrm{~mm}$, are available for tissue retraction. A specially designed fibreoptic clamp allows the use of endoscopes of different angulation and sizes. The light source perfectly illuminates the depth of operating field, and the endoscopic view allows visualization of the procedures on a video screen. This enables nurses and assistants to follow the surgery, facilitating teaching, since the surgeon is the only one with a direct three dimensional view of the operating field. This set up was used in all surgeries.

The size of incision, amount of blood loss, duration of surgery, intraoperative and postoperative complications were recorded. The duration of surgery was noted from time of incision made to closure of the wound of the second stage surgery. The wound was inspected after 48 hours of surgery for any evidence of infection. All the patients were mobilized after 48 hours with Taylor Brace. The patient was discharged with appropriate program of rehabilitation and followed at two weeks for suture removal. The patients were followed at six weeks, three months, six months and one year for assessment of neurological improvement, modification of antitubercular drugs and possible complications.

The data was entered using Microsoft Excel version 8 and analysed using Epi-Info computer programme.

\section{RESULTS}

Data of five patients with minimal invasive decompression for tuberculosis of thoracolumbar junction of spine were collected prospectively. Average age of the patients was 32 years and $80 \%$ were male. A left sided mini thoracotomy was chosen in all the cases. Expandable cages for reconstruction of anterior column were implanted in three cases and tricortical graft in two cases.

The average size of incision was $4 \mathrm{~cm}(4-6 \mathrm{~cm})$. The mean operating time 100 minutes (range 90-120 minutes). The overall mean blood loss in all the cases $400 \mathrm{ml}(300-600)$.

There were no intra or postoperative complications related to minimal access; in particular, there were no vascular or visceral complications. All patients showed minor pain at site of intervention, which disappeared after some days. None of the patients had intercostal neuralgia or post thoracotomy pain syndromes. Postoperative radiograph had shown satisfactory position of cages and graft. Neither postoperative infection nor deep vein thrombosis occurred. Patients were discharged from hospital after an average of seven days (range 3-12). Removal of suture was done after two weeks postoperatively. The table mounted Synframe is shown in figure 1 and use of expandable cage peroperatively in figure 2 . The postoperative anteroposterior radiograph is shown in figure 3 and lateral radiograph in figure 4 .

\section{DISCUSSION}

Minimal invasive spine surgery was introduced in the early 1990 s as an experimental procedure. ${ }^{2}$ The first results of endoscopic spine surgery clearly demonstrated the benefits of these new techniques in terms of reduced surgical trauma and reduced blood loss, with an evident decrease of blood transfusion, less pain at site of operation as well as improved postoperative respiratory function. ${ }^{1,2,8,9}$

The purpose of minimal invasive surgery is not a simple reduction is the size of the skin incision, but rather reducing to a minimum the physical trauma inflicted on the patient, while achieving maximum therapeutic result. ${ }^{12}$ Endoscopic spine intervention do not necessarily achieve the mentioned goal of minimal invasive surgery. 
Karn et al. Minimal Invasive Anterior Decompression in Tuberculosis of ...
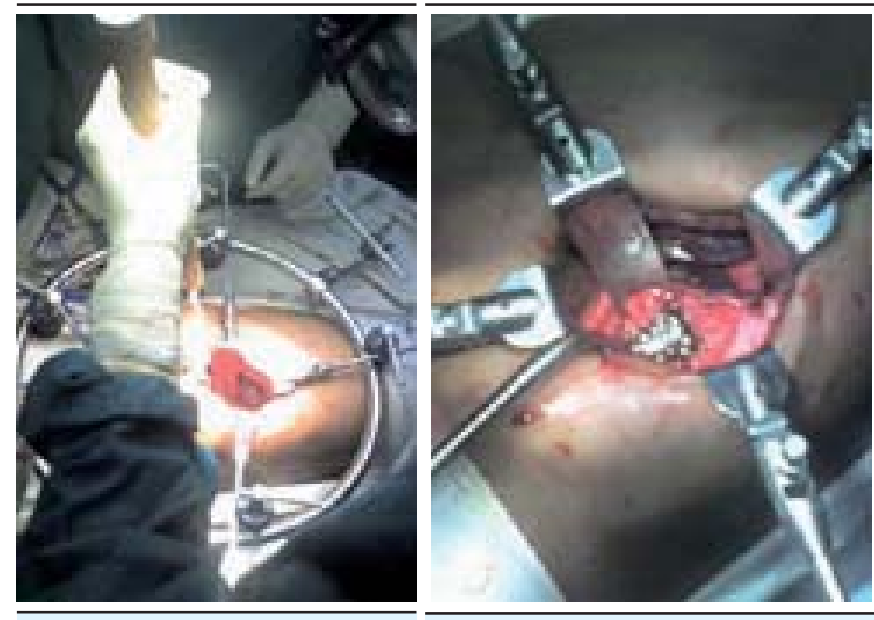

Figure 1. SynFrame
Figure 2. Use of expandable cage

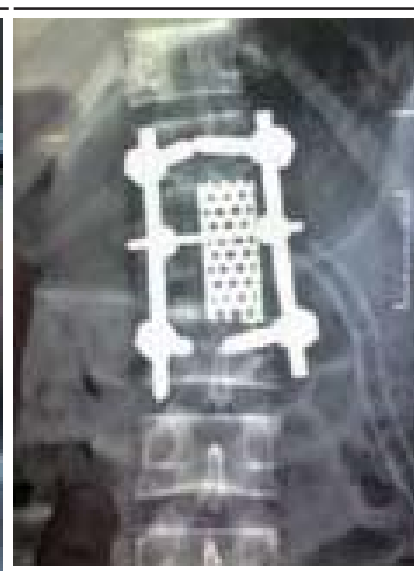

Figure 3. Anteroposterior radiograph postoperatively

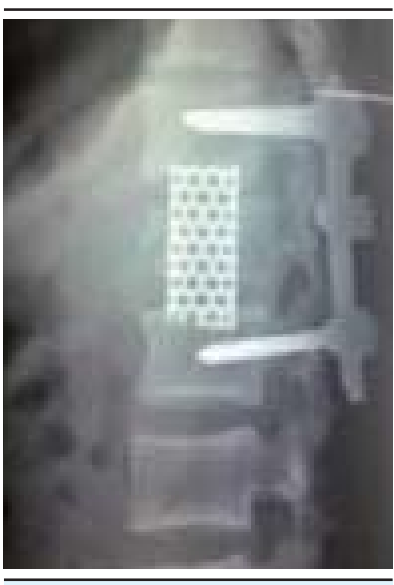

Figure 4. Lateral radiograph postoperatively
Mayer has published a remarkable work about retroperitoneal and transperitoneal approaches using self holding spreader frame. ${ }^{6} \mathrm{He}$ had shown with this method of open but minimal invasive procedure to the lumbar spine, a spinal fusion can be achieved without serious complications. Similar approaches and results have been reported by DeWald et $\mathrm{al}^{13}$ and Thomas et al. ${ }^{14}$

Mayer modified existing retractor systems to the specific needs of anterior lumbar inter-body fusion. However, a universal approach to the spine requires another customized tool, which has been achieved with the development of SynFrame, a retractor system that can be used at almost every part of the spine, for either anterior or posterior approaches.

\section{CONCLUSIONS}

This technology for minimal access spine is safe and easy to use. The ring retractor system allows minimal open surgery to the spine by carrying different devices as well as endoscope, providing an excellent visualization of the operating field, and therefore this novel access technology can be used in all pathologies affecting the spine. The only disadvantage is its high cost.

\section{ACKNOWLEDGEMENTS}

The authors thank paramedics and operating room staff at Paraplegia Hospital, Ahmedabad for their help in completion of the study.

\section{REFERENCES}

1. Rejan JJ, Guyer RD. Endoscopic techniques in spinal surgery. Clin. Orthopedics. 1997;335:122-39.

2. Rejan JJ, Mc Afee PC, Mack MJ, editors. Atlas of endoscopic spine surgery. St. Louis: Quality Medical Publishing; 1995.

3. Dajczman E, Gordon A, Kreisman H, Wolkove N. Long term postthoracotomy pain. Chest. 1991;99:270-3.

4. Faciszewski T, Winter RB, Lonstein JE, Denis F, Johnson L. The surgical and medical perioperative complications of anterior spinal fusion surgery in the thoracic and lumbar spine in adults. Spine. 1995;20:1592-9.

5. Landreneau R, Hazelrigg S, Mack M. Post operative painrelated morbidity. Video-assisted thoracic surgery versus thoracotomy. Ann Thorac Surg. 1993;56:1284-9.

6. Mayer HM. A new microsurgical technique for minimal invasive anterior lumbar interbody fusion. Spine. 1997;22:691-700.

7. Slotman GJ, Stein SC. Laparoscopic L5-S1 discectomy: a cost effective minimally invasive general surgery - neurosurgery team alternative to laminectomy. Am.Surg. 1996;62:64-8.
8. Buhren V, Beiss R, Potulski M. Minimally invasive ventral spondylodesis for injuries of the thoracic and lumbar spine. Chirurgie. 1997;68:1076-84.

9. Olinger A, Hildebrandt U, Mutschler W, Menger MD. First clinical experience with an endoscopic retroperitoneal approach for anterior fusion of lumbar spine fractures from levels T12 to L5. Surg Endosc. 1999;13:1215-9.

10. Thalgott JS, Chin AK, Ameriks JA, Jordan FT, Daubs MD, Giuffre JM. Gasless endoscopic anterior lumbar interbody fusion utilizing BERG approach. Surg Endosc. 2000;14;546-52.

11. Aebi M, Steffen T. Synframe: a preliminary report. Eur Spine J. 2000;9:S44-50.

12. Fitzpatrick JM, Wickham JEA. Minimal Invasive surgery. Br J Surg. 1990;77:721-2.

13. De Wald CJ, Millikan KW, Hammerberg KW, Doolas A, Dewald RL. An open, minimally invasive approach to the lumbar spine. Am Surg. 1999;65:61-8.

14. Kossmann T, Jacobi D, Trentz O. The use of retractor system (SynFrame) for open, minimal invasive reconstruction of the anterior column of the thoracic and lumbar spine. Eur Spine J. 2001;10:396-402. 